\title{
Building a Process Driven Organization with the Process Normalization Technique
}

\author{
Václav Řepa* \\ Department of Information Technologies, Faculty of Informatics and Statistics, \\ University of Economics, Prague, Czech Republic \\ repa@vse.cz
}

\begin{abstract}
Despite the importance, the methodological standards for business process modeling are still very much insufficient. One of the most important aspects which must be covered with the process models is essential unity of object oriented and process oriented views of a business system. To achieve such unity, this article introduces a process normalization technique as part of the Methodology for Modeling and Analysis of Business Processes (MMABP). This technique is freely inspired by the famous 'ancient' Normalization of Data Structures technique which we regard as relevant and correct because of the essential unity of objects and processes in the business system. At the same time it can be seen that harmonizing the structure of processes with the (conceptual) structure of the Real World directly fulfills the objective. The goal of this article is to illustrate the steps of process normalization with examples. Important connected problems of structural algorithmic thinking, as well as other ways of manifesting the previously mentioned essential object-processes unity in the business system, are also discussed in the article.
\end{abstract}

Keywords: Business process model, Normalization, Structured programming, Object orientation, Process orientation

\section{Introduction}

Despite their importance, the methodological standards for business process modeling are still very much insufficient. An example of insufficiency is seen in the modeling standard, Business Process Model and Notation (BPMN) [1]. Besides its serious internal contradictions, BPMN is still able to cover just a small part of the needed contents of the business process model. One of the most important aspects which needs to be covered by the process models, is the essential unity of object oriented and process oriented views of a business system.

The ideas expressed in this article are based on the Methodology for Modeling and Analysis of Business Processes - MMABP [2]. The first basic principle of this methodology is the Principle of Modeling, which expresses the presumption that the objective basis for the implementation of

\footnotetext{
* Corresponding author

(C) 2018 Václav Řepa. This is an Open Access article licensed under the Creative Commons Attribution License (CC BY 4.0), https://creativecommons.org/licenses/by/4.0

Reference: V. Ǩepa, "Building a Process Driven Organization with the Process Normalization Technique," Complex Systems Informatics and Modeling Quarterly, CSIMQ, no. 14, pp. 22-37, 2018. Available: https://doi.org/10.7250/csimq.2018-14.02

Additional information. Author's ORCID iD: V. Řepa - orcid.org/0000-0001-9113-3447. PII S225599221800081X. Received: 20 January 2018. Accepted: 6 April 2018. Available online: 30 April 2018.
} 
the business system in the organization must be constituted by real facts existing outside of, and independently of, the organization. In other words, every organization is actually the implementation of some business system (business idea) and must be based on a model of the relevant part of the Real World.

MMABP distinguishes between two basic dimensions of the Real World: structure (object view) and behavior (process view). In both of these dimensions there are two basic types of model: global (system) view on the system as a whole and detailed (particular) view on just one element of the system. The main difference between the global (system) and the detailed (particular) view is the factor of time. The system (global) view always tries to abstract this factor and then focus on stable, time independent, aspects of the modeled system, while the detailed model respects the time factor by focusing mainly on it. The global view, then, can be characterized as object-oriented and the detailed view as process (algorithmic)-oriented (see Figure 1).

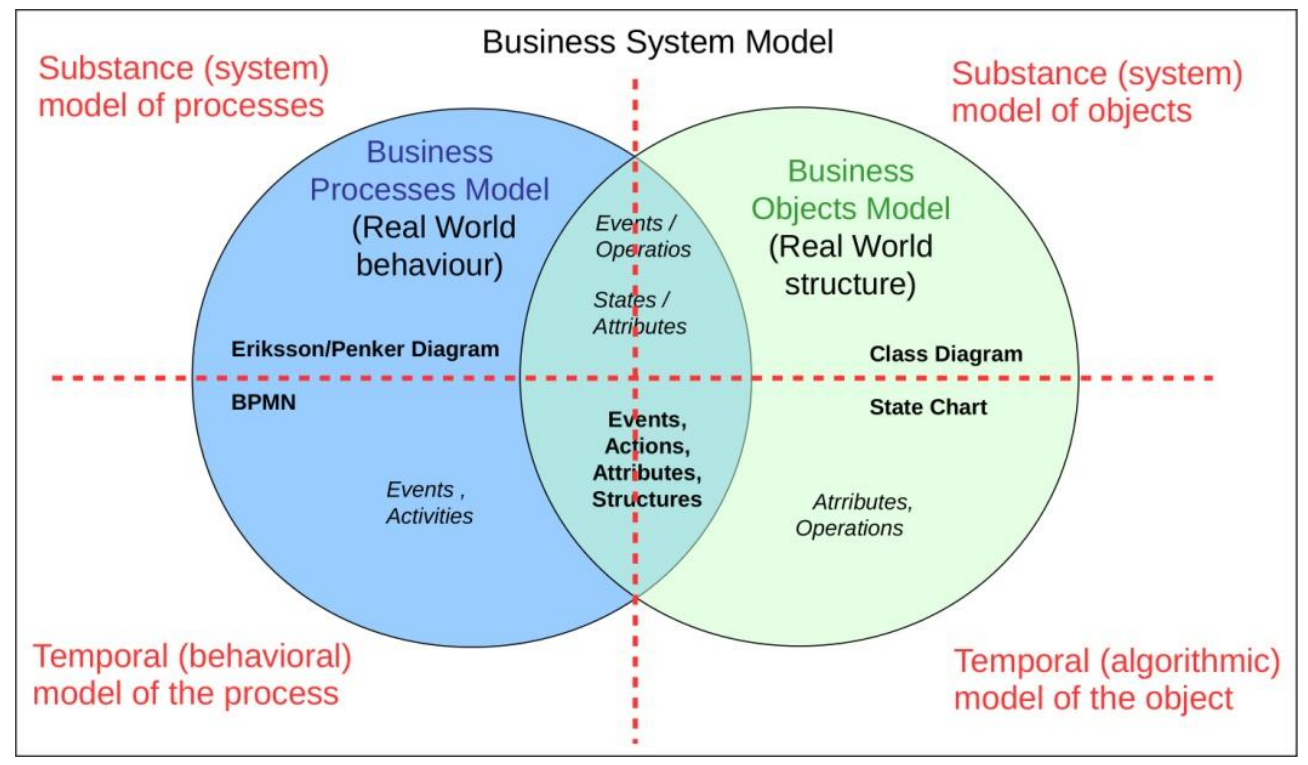

Figure 1. Two basic types of model in two dimensions of the Real World Model

In this context MMABP distinguishes between the concepts: business system and business process system. Business system is a system (i.e. a set of elements and their mutual relationships) in any domain of the Real World which is influenced by human activity. Business process system is a system of business processes, i.e. a view of a business system reduced purely to business processes; alternatively a set of sets of intentional activities, each representing the way of achieving some goal. Consequently, we can also speak about the business object system as a system of business objects, which represents another reduction of the business system: a set of objects and their mutual relationships representing a general modality of the Real World (alias 'business rules'). So the business system is a complex entity which we can view from two basic perspectives: as a system of business rules (business object system) and as a system of business activities (business process system). For a full understanding of the business system in its complexity, we always have to look at the system from both of these perspectives.

Sufficient business system modeling methodology has to support both types of models (global vs. detailed) in both dimensions (structural vs. behavioral). Moreover, both dimensions always have to take into account their mutual interconnections. At the same time, the methodology also has to integrate both essential components of a business system: its managerial and technology elements. The proposed Process Normalization technique tries to cover all those aspects by offering a certain way of integration of the global process map with the process details, 
intentional processes with conceptual objects, and also the process structure with its managerial meaning.

This article is an extension of the earlier published paper [3]. It is divided into five main sections. In the Section 2, following this Introduction section, we explain the crucial ideas from the field of business process management, which the idea of process normalization is based on. The natural difference between the key and support processes is explained there, together with the importance of distinguishing between them. Section 3 is the main section of the article in which we describe the Process Normalization technique. First, the ideas of Normalization of Data Structures, as a main inspiration for the Normalization of Processes are described, generalized and interpreted in the field of business processes in the form of the goals of the Normalization of Processes. In the following two sub-sections the procedure of the Normalization of Processes is described and explained with the use of a real world example. In Section 4 we discuss important aspects, related to the Normalization of Processes, such as reengineering features of handling the business processes, general theory of structures and natural close relationships between the structure of business processes and the structure of business objects. In the Conclusions section we summarize the purpose and practical meaning of the Process Normalization technique, its potentials and limitations and outline a proposal for the needed future work.

\section{Business Process System and Its Managerial Context}

By the 'business process system' we mean the system of mutually collaborating business processes. To understand the 'business essence' of the collaboration of processes in terms of ideas of process-driven management [4] one primarily has to differentiate between two basic functional types of processes: key processes versus support processes. As customer needs are constantly changing, the processes in the organization should change as well. This means that any process in the organization should be linked, as directly as possible to the customer needs. The general classification of processes in the organization is reflected in Table 1:

- Key processes are those processes in the organization which are linked directly to the customer, covering the whole business cycle from expression of the customer need to its satisfaction with the product/service.

- Support processes are processes which are linked to the customer indirectly - through key processes which they are supporting with respect to particular products/services.

Table 1. Key versus Support Processes

\begin{tabular}{|c|l|l|}
\hline Customer's needs & \multicolumn{1}{|c|}{ Key process } & \multicolumn{1}{c|}{ Support process } \\
\hline Responsibility & $\begin{array}{l}\text { Management - oriented. } \\
\text { Responsible primarily for the } \\
\text { context of the whole business case } \\
\text { from the customer point of view. }\end{array}$ & $\begin{array}{l}\text { Fulfilled indirectly, through the } \\
\text { support of other processes }\end{array}$ \\
\hline $\begin{array}{c}\text { Production - oriented. } \\
\text { service, not for the context in which } \\
\text { it is used. }\end{array}$ \\
\hline Dynamics & $\begin{array}{l}\text { Very dynamic, often changing } \\
\text { permanently developing. }\end{array}$ & $\begin{array}{l}\text { Stable, no need for changes, offering } \\
\text { standardized services. }\end{array}$ \\
\hline
\end{tabular}

Table 1 shows the comparison of the essential characteristics of two basic types of processes. It can clearly be seen that these two types of processes are substantially different from one another. The comparison is made from three points of view: how the process fulfills the customer needs, for what the process is responsible, and how much it is naturally dynamic. 
The value of the key process is given by its direct impact on the value for the customer as that is its main goal. The values of other (support) processes derive from the services by which these processes support other processes. In this way, every process is ultimately connected to the value for the customer either directly (key process) or through its services performed for other processes. Key processes thus represent a specific enterprise's way of satisfying the customer needs while support processes represent rather standard functionality often connected with some type of technology. Consequently, key processes are very dynamic, often changing, permanently developing. Every instance of the key process differs from other instances and often brings new ideas for the further development of the business. Support processes are mostly static, stable, offering standardized services, and multiply usable without a need to change them from instance to instance.

So the main effort in the process of creating the conception of the system of processes must be establishing the equilibrium of needed dynamics of key processes, on the one hand, and the necessary stability of the system ensured by its maximally standard support processes, on the other.

\section{Process Normalization}

Normalization of processes is a technique freely inspired by the Normalization of Data Structures technique initially introduced by E.F.Codd in [5], then elaborated in further detail by R.F.Boyce in [6] and comprehensively explained in [7]. Although Codd's original intention was mainly technical in terms of a database system design, this technique started revealing the essential Principle of Modeling in the field of information systems development, which has later been defined by P.Chen in [8]. We regard the Principle of Modeling as essential and valid in all dimensions. All kinds of models in the field of information system development are, in some ways, models of the Real World; even including business process models. Regarding this fact, together with the aforementioned essential unity of objects and processes in the business system, as it is defined in MMABP [2], we can conclude that the same essential principles, which the Normalization of Data Structures is based on, are also valid for the proposed Normalization of Processes. As business processes, seen from the system point of view (i.e. as a system of mutually collaborative processes), are, in general, special kinds of objects, then also the structuring of them should follow the general rules for structuring objects. So the goals of the Normalization of Processes can be defined as follows:

(a) Reduce redundancy of process activities.

(b) Ensure that all activities and non-initial events are dependent on the initial event in terms of the process goals.

(c) Eliminate unnecessary hidden dependency relationships within a process.

The interpretation of the Processes Normalization goals is focused mainly on the support of basic ideas of process-driven management excellently expressed in [4].

Goal (a): redundancy of process activities means unnecessary repeating of activities, with essentially the same content and meaning, in different processes. Unrecognized redundant occurrence of activities with the same contents in different processes, is one of the typical consequences of hierarchical organization mentioned by Hammer in [4] as a 'symptom of broken processes'. Eliminating the redundancy of activities leads to the creation of such a system of mutually collaborating processes, wherein one essential activity has its own stable place in exactly one process. All redundant occurrences of the activity in various processes are then replaced by the service from the 'home' process of this essential activity. Generalizing this situation, one can see that each redundant use of a particular activity points to the essential 
relationship between two processes: the original one (supported) and the supporting one. This kind of the collaboration of processes directly corresponds to the fundamental meaning of socalled 'support process' as characterized in the previous section.

Goal (b): unconditional clear dependency of all activities on the initial event represents the natural specialization of processes. This essential feature of the process driven organization explains the natural meaning of specialization in the field of management. It replaces the traditional hierarchy-based way of the specialization of management where, in fact the, higher level of management means, at the same time, the lower level of specialization. In this way the manager loses contact with the factual meaning of what he manages. The higher the level of management, the worse this situation is. It is obvious that there must exist a certain level of hierarchical position at which the manager completely loses any direct link with the factual meaning of the company's performance; and is forced to abstract it to mere statistical indicators. Then the only means for management, i.e. making the crucial decisions, which remain to him, are financial means. Management of the organization, oriented in such a way is, in influential literature, usually regarded as opposite to process-oriented management [4], [9]. The solution offered by the process orientation is based on the change of the subject matter on which the managerial effort should be focused. Instead of hierarchical aggregating the whole subject of management up to unmanageable units, in process-oriented management is based on strongly distinguishing between the managerial and the factual aspects of the work in the organization. The process description focuses on its managerial aspects, i.e. watching circumstances and decision making, while the factual aspects of the work are hidden in the productive activities of the process. If the productive activity is more complex, i.e. it contains some necessary decision points which require managerial effort, it should be implemented as a service from another supporting - process. Such collaboration of processes represents the division of responsibility for management. In this way the responsibility of the process manager can be concentrated on the management of activities in terms of achieving the process goals and thus be relieved of the responsibility for embedded activities. Responsibility for the process activities naturally belongs to those who perform them. Depending on the complexity of the activity it can be performed as a simple action or as a service from another process. In both cases there is a facilitator, different from the process manager, responsible for the given activity: either the performing of the activity itself or the supporting process, no matter whether it is performed by human or machine, or if it is outsourced.

Such a form of the division of responsibility among the business actors requires focusing of each process on an exactly defined clear goal, representing the essential meaning of the process. All process activities have to have a unique role in achieving the process goal, which is defined by the starting event of the process.

The goal (b), together with goal (c), support the principle of key processes as centerlines of the final meaning of all activities in the organization which represents the definite way of ensuring the relationship to the customer-oriented value of the performance of the company, as a main principle of process orientation [4], [9].

Using the Process Normalization technique, the key processes are relieved of all activities which signalize the existence of possible process goals, other than the primary process goal expressed by the dependency on the initial event. These supportive process sub-goals usually signalize the existence of other, more general, sub-processes which are focused on some specific goal and are hidden in the body of the normalized process. From the point of view of the goal of the normalized process, these processes represent the set of supporting actions, with more general meaning, which are needed as a step on the way to the final process target. Their general meaning is, then, the reason for their removal from the body of the normalized process and establishing them as standalone support processes. In this way the normalized process is freed of all non-essential activities which are removed to more general support processes and the 
necessary relationships, between the original process and the new ones, are uncovered in terms of the meaning of support activities in the context of the goal of the normalized process.

Consequently, the input assumptions for the normalizing procedure are as follows:

- The logical process represents a part of the Real World consisting of natural process chains and their relationships.

- Each activity in the process represents an activity from some natural process chain or relationship among process chains.

- Each event in the process represents an activity of some external actor or related (collaborating) process chain.

- Each natural process chain hidden in the logical process can be uniquely identified by some event or by a logical structure of events. Such event (structure of events) is called an 'initial event'.

\subsection{The Procedure of Process Normalization}

The procedure of process normalization is defined as a sequence of steps by particular Normal Forms. The initial condition for each step is that the process is in the previous Normal Form (i.e. fulfills its required characteristics). Particular Normal Forms are defined as follows:

\section{1st Normal Form (iterative generalizable structures free)}

The process is in the 1st Normal Form if the bodies of all of its repeating non-elementary structural parts (iterations) have been removed to standalone processes and replaced by process states. Each removed part of the process has been identified with the corresponding business system object. Its starting event has been defined as a request from the original process. Its product has been defined as a service and corresponding event from the point of view of the original (i.e. receiving) process.

\section{2nd Normal Form (alternative generalizable structures free)}

The process is in the 2nd Normal Form if it is in the 1st Normal Form and the bodies of all of its mutually alternative non-elementary structural parts (selections) have been removed to standalone processes and replaced by process states. Each removed part of the process has been identified with the corresponding business system object. Its starting event has been defined as a request from the original process. Its product has been defined as a service and corresponding event from the point of view of the original (i.e. receiving) process.

\section{3rd Normal Form (parallel generalizable structures free)}

The process is in the 3rd Normal Form if it is in the 2nd Normal Form and the bodies of all of its mutually parallel non-elementary structural parts (simultaneities) have been removed to standalone processes and replaced by process states. Each removed part of the process has been identified with the corresponding business system object. Its starting event has been defined as a request from the original process. Its product has been defined as a service and corresponding event from the point of view of the original (i.e. receiving) process.

\section{4th Normal Form (hidden generalizable sub-structures free)}

The process is in the 4th Normal Form if it is in the 3rd Normal Form and the bodies of all of its non-elementary structural parts (sequences), which are not fully specific to the starting event of the process, have been removed to standalone processes and replaced by process states. Each removed part of the process has been identified with the corresponding business system object. Its starting event has been defined as a request from the original process. Its product has been 
defined as a service and corresponding event from the point of view of the original (i.e. receiving) process.

From the point of view of the business process system the Process Normalization can be regarded as a way of uncovering the natural supporting processes which are hidden in the body of the given process. In the first step all repeatedly used support services are uncovered in the process. The second step uncovers all conditionally used support services and the third step uncovers all substantially different (parallel) services hidden in the same process step. In the last step all remaining parts of the process, which can be also regarded as standalone services, are uncovered. The ordering of steps is important for similar reasons to those in the normalization of data structures. To be able to correctly interpret alternative sub-structures (branches) in the process one has to remove all repeated structures of activities first (1st to 2nd Normal Form) as the decision about the repetition (end of the loop) might be incorrectly interpreted as a fork, signalizing mutually alternative sub-structures. Seeking for parallel structures does not make sense between different alternative structures, so the structure has to be in the 2nd Normal Form before it can be transformed to the 3rd Normal Form. Similarly, an identified sub-structure cannot overstep the border between alternative and parallel branches of the process. These rules generally follow from the theory of structured thinking most comprehensively described in [10] and [11]. Relationships of the process normalization technique to this theory are also discussed in the Section 4 of the article.

\subsection{Example of Process Normalization}

The notation for the detailed process structure used in the example is a strong reduction of BPMN [1] used by MMABP methodology as it follows on from the business process metamodel [2]. To make BPMN methodologically consistent with the MMABP principles, it has to be reduced on just the basic set of language constructs: activities, two essential types of events (ad-hoc and timer), decisions and logical connectors, and process inputs and outputs; represented in BPMN as associated "data objects". Events are defined as always being external influences of the process. The concept of process state, which is completely missing in the BPMN, is compensated by the AND join construction, expressing the meaning of the process state as a necessary synchronization of the process flow with the external influence (set of events). A more detailed explanation of the need to improve BPMN can be found in [12].

According to the MMABP the detailed model of the process structure has to be accomplished with the contextual view of the whole business process system, the so-called Process Map. MMABP uses the widespread Eriksson/Penker notation [13] for this model.

Figure 2 shows un-normalized process Customer Order Management in the BPMN [1] notation. The process description approach follows the MMABP methodology [2] especially paying attention to communication with external collaborators. In this un-normalized form the process collaborates only with the customer. See the process states ("parallel gates" in BPMN) at the beginning (negotiation of the order) and before the end (payment) of the process.

To transform the process to the first Normal Form we have to identify all repeating substructures in the process structure. As we work with an algorithmic structure we are seeking for so-called iterations or cycles in the algorithm. There are three repeating sub-structures in the Customer Order Management process, each connected with some objective concept. The process starts with the repeating negotiation with the Customer about the contents of the Order. The next repetition in the process represents the series of Production steps. The third repetition again represents the communication of the process with the Customer, this time about the Payment. 


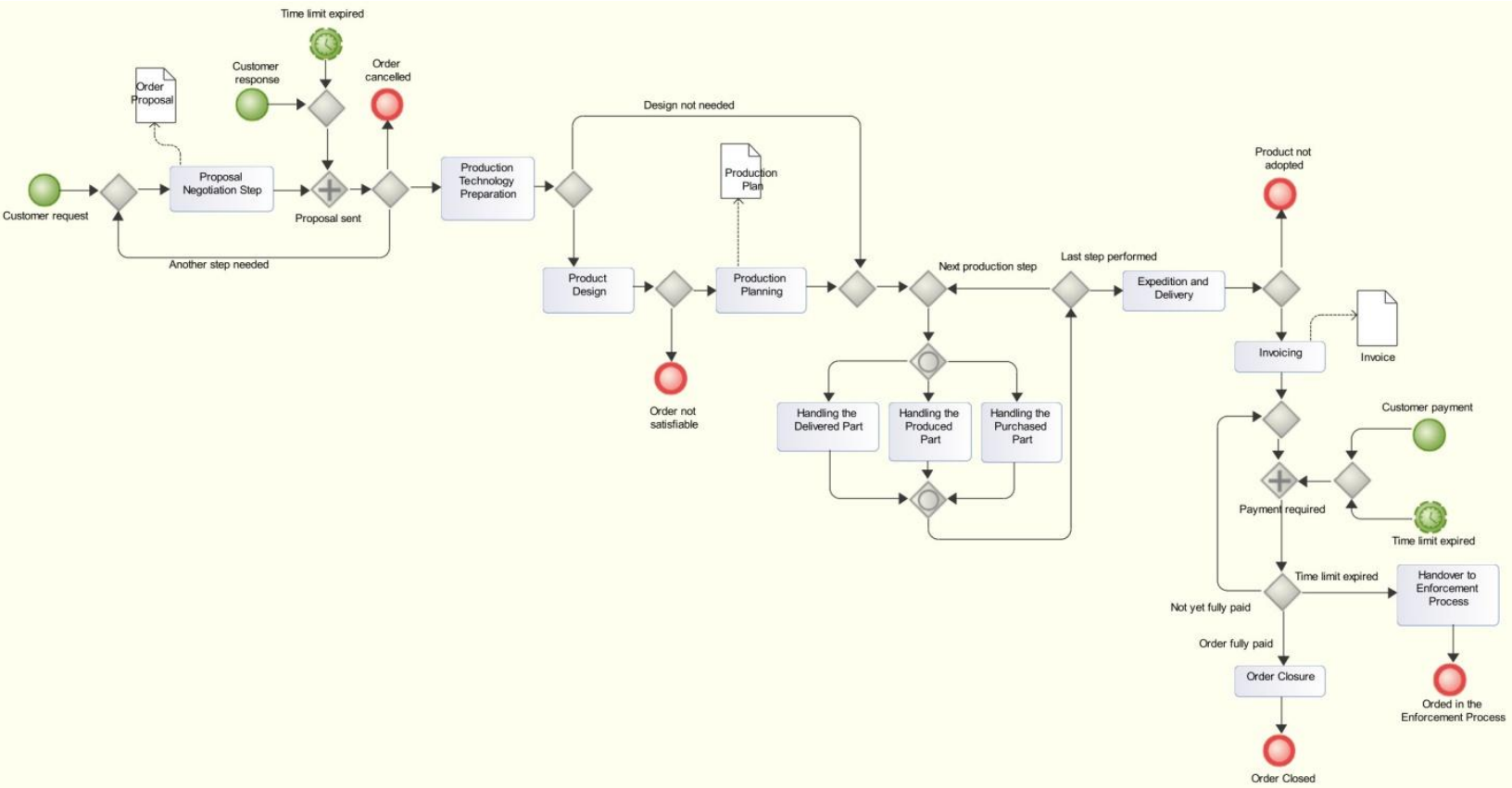

Figure 2. Example - Un-normalized process Customer Order Management

Figure 3 shows the changes in the process caused by transforming it into the first Normal Form. Repeating parts which represent handling the objects Order, Production and Payment have been identified, removed to standalone support processes, and replaced by the new process states; each representing waiting for the external service.

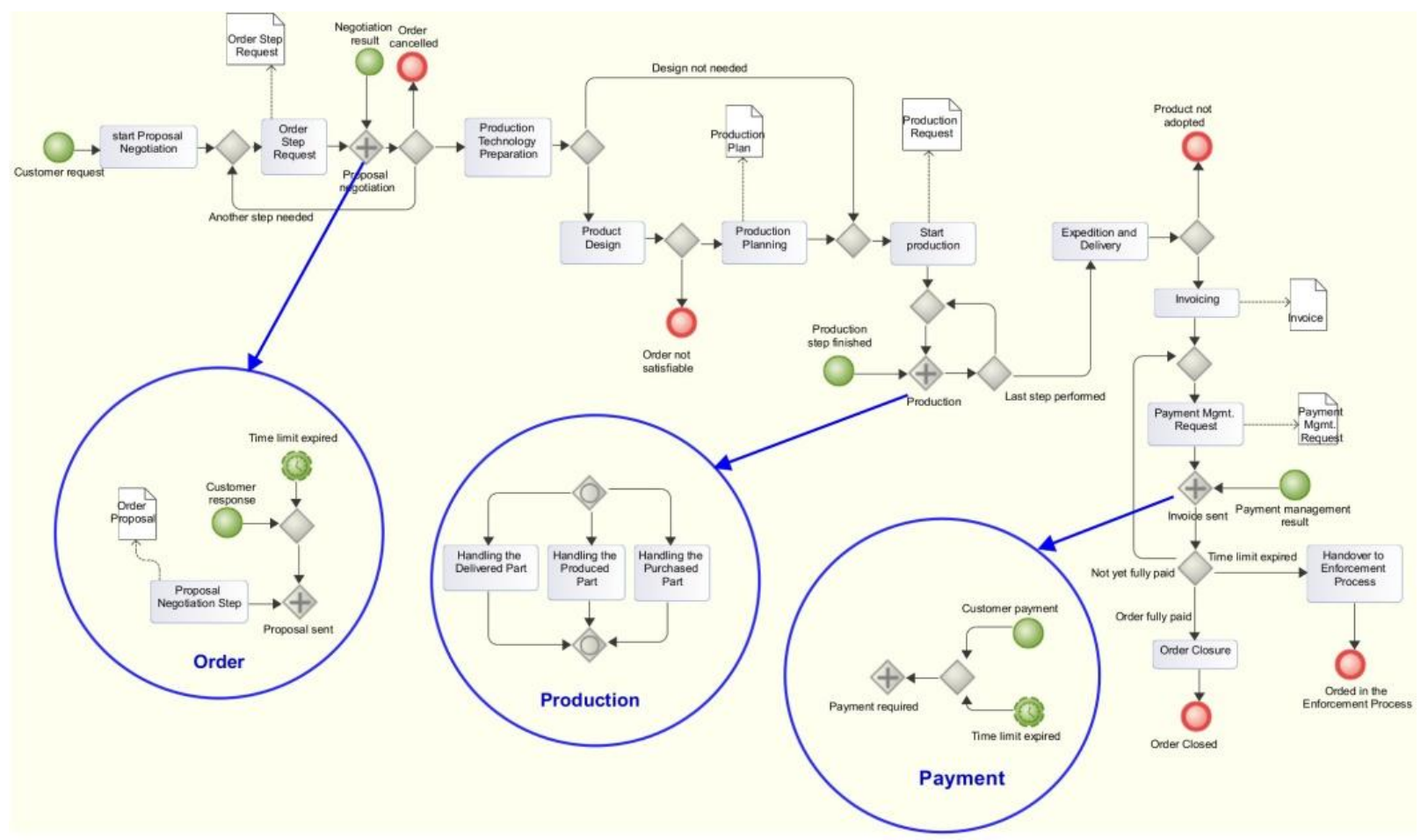

Figure 3. Example - Process in the 1st Normal Form

The process map in Figure 4 shows the sequential changes in the process system. Un-normalized process represents the existence of just one process, which covers all activities 
necessary for achieving the process goal without any help from other processes. The only external actor communicating with the process is Customer. Transforming the process to the 1st Normal Form causes the creation of three new processes collaborating with the original process: Order Step Management, Production Step Management and Payment Step Management. Now it is possible to start speaking about the system of processes instead of just a single process covering everything. The manager of the Customer Order Management process is not responsible for the negotiation of the order details, handling the customer's payment, nor organizing the production cycle; this responsibility lies with the managers of supporting processes. The manager of the Customer Order Management process can now better focus on the goal of his own process - delivery of value to the customer. Besides the generally positive effect of the specialization, such division of the work significantly contributes to the needed standardization of the enterprise functionality. It is obvious that all three newly created processes are more general than merely being regarded as special services for just this specific supported process. This allows exploiting the maximum effect of the specialization up to the ultimate solution in the form of complete outsourcing of the process. Moreover, depending on the 'automatability' of the process' subject of specialization, in this way the system of processes in the organization can be harmonized as much as possible with the standard ERP functionality together with keeping up the needed flexibility of the information system.

Unlike the negotiation about the order or payment handling, the production cycle in the initial proposal of the process (un-normalized process) does not represent the communication of the process with an external actor. It is clearly an internal issue of the process. Removing this structure to the standalone support process caused a new state in the original process which now represents waiting for the external service. Also, creation of new support processes Order Step Management and Payment Step Management brought new states into the original process, together with removing both former states, which are now parts of the new support processes.

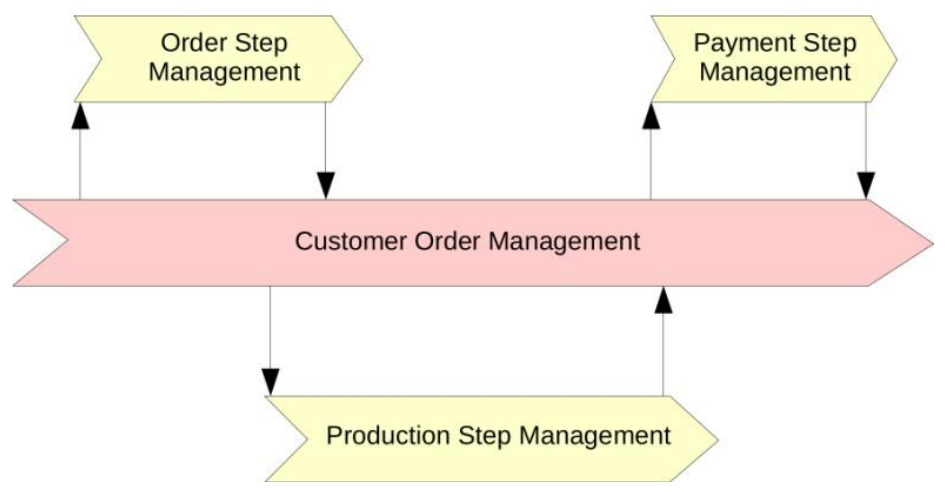

Figure 4. Process Map (1st Normal Form)

The process at Figure 5 is in the 2nd Normal Form, after the removal of two conditional parts which can be regarded as standalone standard support services. Product Preparation is performed only if the customer requires a nonstandard product and the Order Finalization branch, only if the customer adopted the delivered product. As there are no remaining parallel parts in this process it is also in the 3rd Normal Form (see Figure 5). 


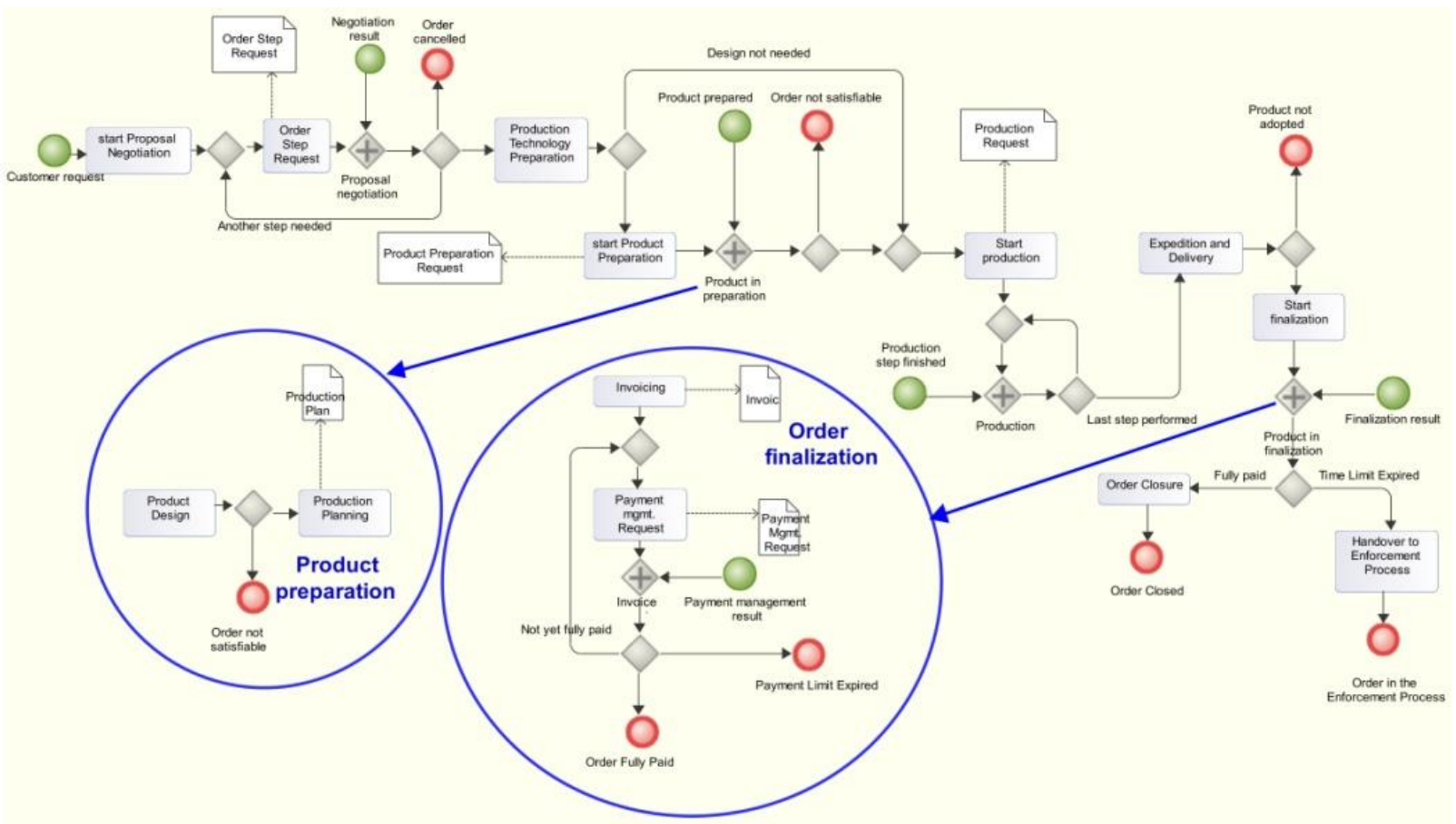

Figure 5. Example - Process in the 2nd and 3rd Normal Forms

The process map at Figure 6 shows the system of processes after transforming the key process to 2nd (and 3rd) Normal Form. The new support processes have been created: Product Preparation and Order Finalization. As the support process Order Finalization also contains communication with the already existing support process Payment Step Management, the key process no longer directly communicates with this process, which means that the quality of this service is now a function of the relationship between the Payment Step Management and Order Finalization processes.

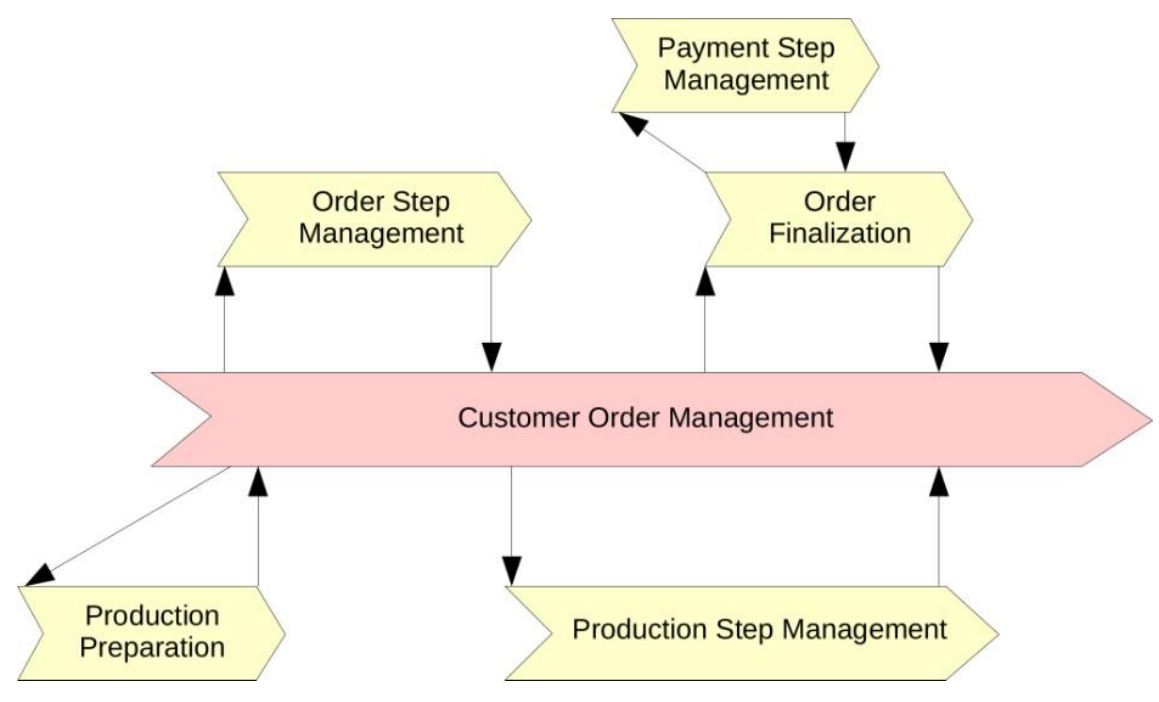

Figure 6. Process Map (2nd and 3rd Normal Forms)

In the 4th Normal Form (Figure 7) the process has been subjected to the removal of two parts representing standalone standard services: Production Technology and Expedition\&Delivery. All remaining parts of the process are then fully specific to the starting event Customer request. 
Corresponding changes resulting from placing the process into the fourth Normal Form in the system of processes are visible in the Process Map at Figure 8.

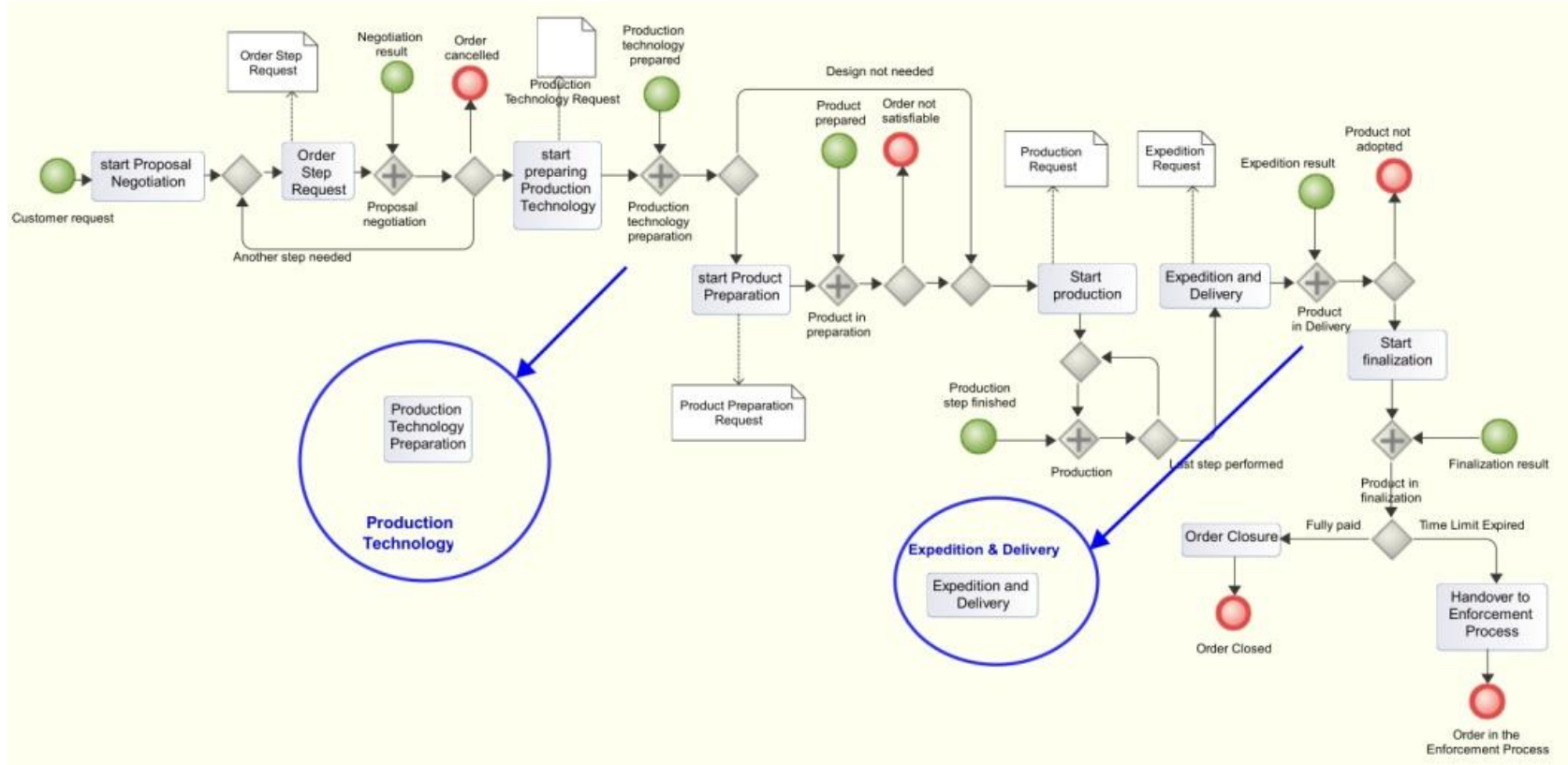

Figure 7. Example - Process in the 4th Normal Form

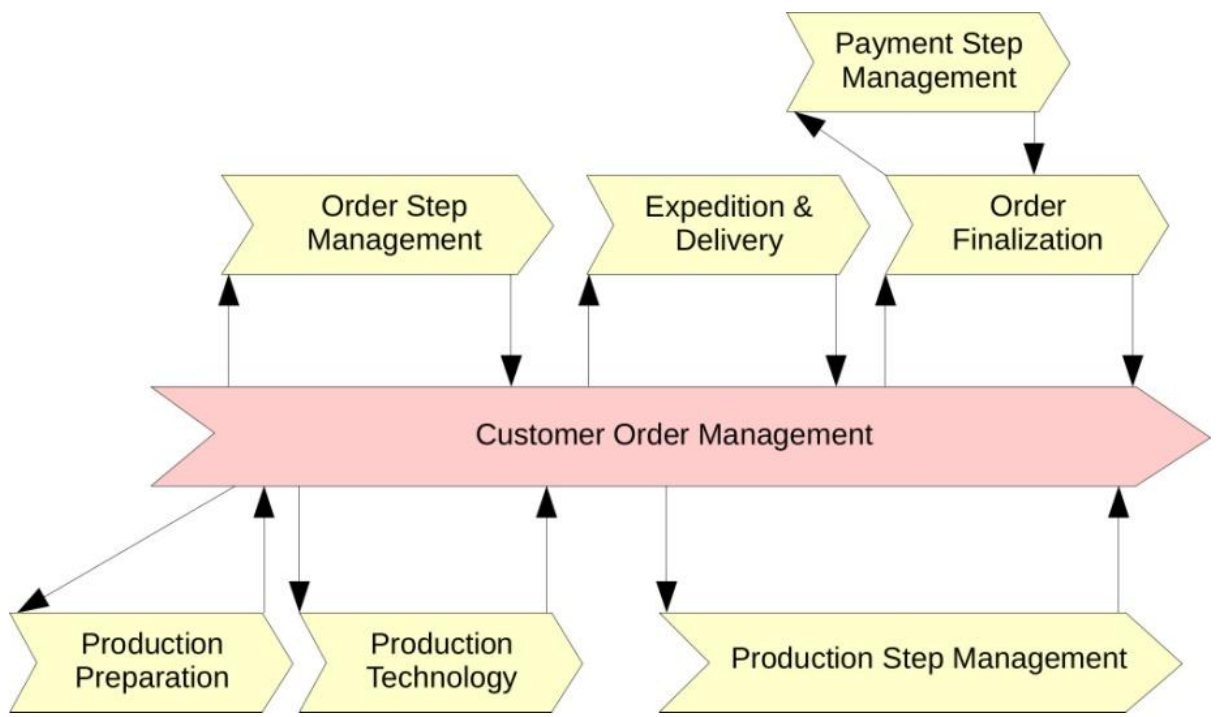

Figure 8. Process Map (4th Normal Form)

Figure 9 shows the resulting map of processes, after the full normalization of the whole system of processes, which started with just the normalization of the original key process Customer Order Management. Besides the newly created support processes, discussed in the previous comments about the particular Normal Forms of the process, there are also several other new processes created by the normalization of new support processes: Product Design and Production Planning which were removed from the Production Preparation support process, and three former parallel parts of the Production Step Management process. 
The final Process Map shows the structure of mutually collaborating processes following the common goal - the final product of the key process Customer Order Management. The structure perfectly reflects the main characteristics of the process driven organization:

- All processes are organized in the network structure, which means that they are generally independent, and their relationships reflect exactly their collaboration: mutually supporting each other with the services. There is no general hierarchy of processes.

- The common meaning of all processes in the map is revealed by the key process which is the final consumer of the supporting services, thus its final product defines the meaning of the presence of all processes in the map.

- Such a structure of generally independent processes which still follow the common goal, can be called a purpose-centric collaboration network structure.

- The process in the center of the structure (key process) represents the direct contact with the Customer, a representative of the common purpose of the whole structure. As the main idea of process-driven management is to make the organization flexible towards the customer needs, the central (key) process is naturally the most dynamic and specific (purposeoriented) process in the structure.

- Consequently, with other (support) processes, the greater their distance from the center of the structure, the more they are universal, generally oriented, and 'static' in terms of requiring no special need for their maintenance. For instance, see in Figure 9 the 'first order' support processes Product Preparation, Production Step Management and Order Finalization which are obviously closer to the specific customer aspects than to the more general 'second order' support processes like Product Design, Purchase of Part or Enforcement Process.

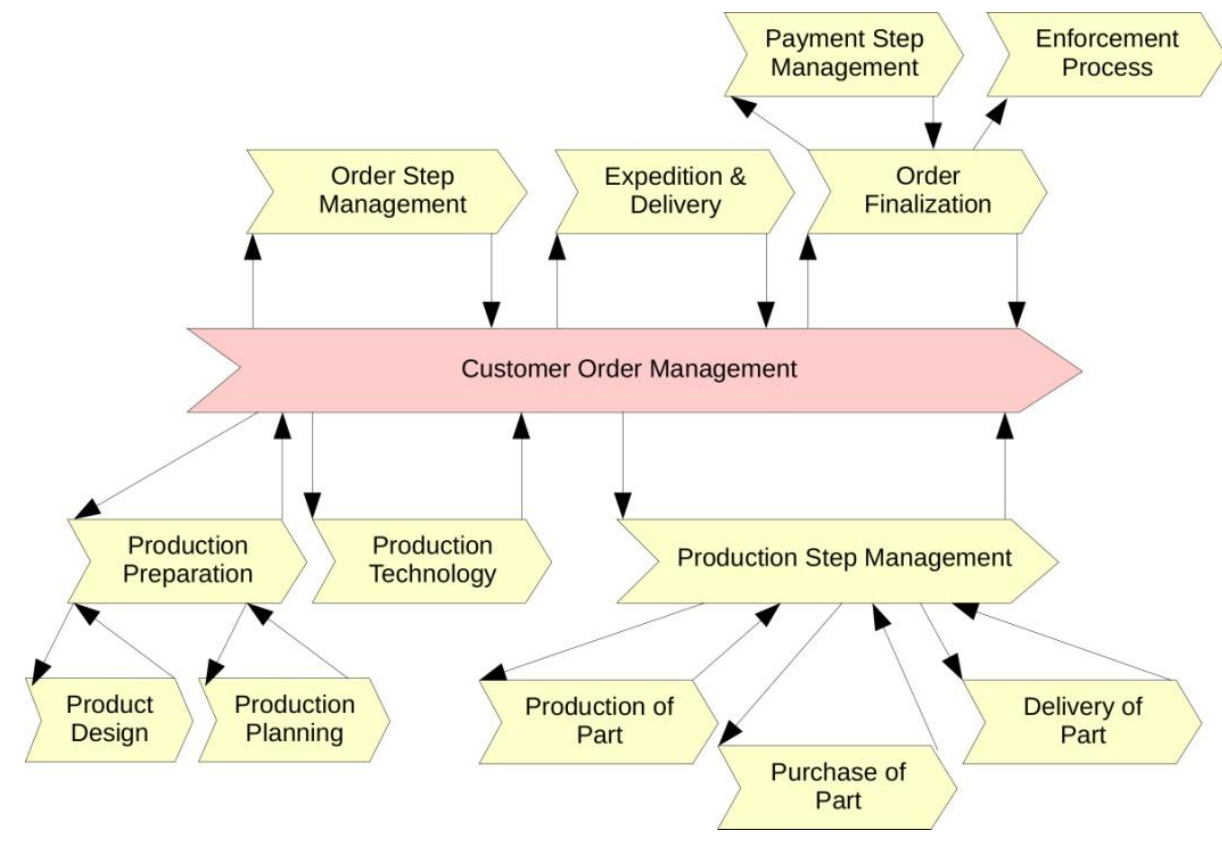

Figure 9. Final Process Map

\section{Discussion}

The essential meaning of the Process Normalization can be characterized as a summarization of the needed decisions and their mutual dependencies, led by the intention to distinguish, as much as possible, between the individuality of the normalized process and the natural generality of the processes which support it. Such principal differentiation is a way that is certain to achieve the 
needed equilibrium of the maximal flexibility on one hand and maximal stability of the enterprise performance on the other hand; every necessary process which does not directly fulfill the primary function of the organization should be stabilized as much as possible, up to its complete outsourcing. In this way, the process outsourcing can be regarded as the boundary of the maximal possible stabilization of the process. The often discussed automation of processes, then, should be regarded as a special case of outsourcing, where the 'service supplier' is a machine. A significant outcome of this short reflection, is the fact that the complete automation of the enterprise, including the automation of key processes, which is often regarded as a finite goal of Business Process Re-engineering, is clearly nonsense, as it is in contradiction with the main idea of the process driven management [4]. Once the process is outsourced, it cannot any more be regarded as a process in terms of the organization's performance. From the organization's point of view, that original process then exists just as a single service delivered by the external supplier, which means that the organization is no longer responsible for its performance, as it now plays the role of the customer.

The crucial condition for the proper use of Process Normalization is the ability to think about the process in a structural way, which means to be able to see the process in terms of the hierarchical structure of sequences, selections and iterations as is defined in [10] and generalized in [11]. The Structure Diagram introduced by Jackson in [10] is the best tool for the description of the process in such a way. Figure 10 shows the description of the Customer Order Management process by means of the Structure Diagram. Nevertheless, a structural viewing of a business process for the purpose of the Process Normalization technique also requires expressing possible parallel actions in the process. For this purpose we enrich the original concept of three basic structure types (sequence, selection and iteration) from [10] by the fourth type 'simultaneity' which represents the parallel running of actions synchronized at the beginning and at the end. In the Jackson's Structure Diagram we label the elements of this additional structure with the plus sign ('+') in the upper right corner (see Figure 10). The structural view of an algorithm in general prevents parallelism as it is a determinant of the boundary between a single algorithm and the system of cooperating algorithms (see [11] for instance). Despite this, the parallelism can be accepted as a fourth basic type of structure if it is controlled: a simultaneity can be used only if beginnings as well as ends of all parallel actions are synchronized at a single point of time, which allows the regarding of the whole structure as a single element, from the point of view of the upper level of the structure. Such encapsulation of parallel actions in a single structure element, prevents the possible corruption of the nature of an algorithm ${ }^{\dagger}$. Another complement to the Jackson's Structure Diagram is the predefined action 'End' which means the termination of the process before its regular end. Use of the 'End' expresses the situation when the process has to be terminated for some specific objective purpose at the particular point of its structure; an exception from the regular process flow. Therefore, the End operation can be used only as a part of a selection structure under the condition which represents the specific objective reason for the termination of the process. Usually, such a situation should be expressed in the Structure Diagram as an upper-level selection in order to maintain the principle that the structure has just one end. From the point of view of the time flow, such a description, however, expresses the decision before the action which allows this decision. The Jackson's method generally solves this problem with a special technique called 'backtracking' [10] which allows the necessary exceptional jump from the structure in the implemented program, together with maintaining the

For instance, Tse in [14] considers even five basic structure types: 'sequence, selection, parallelism, iteration and procedure call' and mentions also the rule that 'every selection or parallelism must have a single input state and a single output state' [14]. It can be seen that the Tse's 'procedure call' represents in fact any sub-structure invoked by the 'call' operation, and that the same is implicitly ensured in Jackson's diagram with its hierarchical nature. The simultaneity thus remains the only natural structure type not covered by the original Jackson's diagram. 
clearly static 'conceptual' description of the program structure. Nevertheless, for the purpose of the Process Normalization we need to describe the process in a structural way, but also as a process, respecting primarily the time flow, which is the main reason for this complement to the original Structure Diagram.

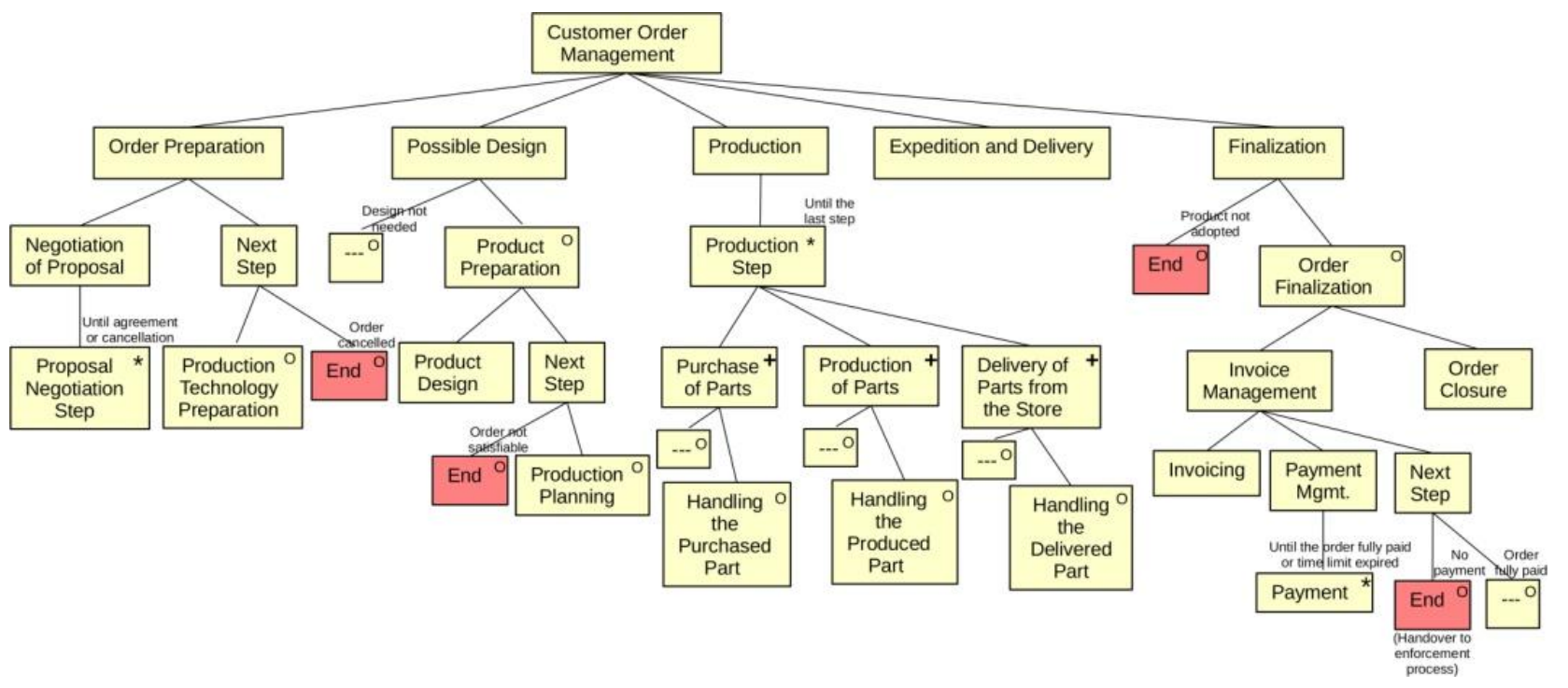

Figure 10. Structural view of the process

Process Normalization is also closely connected with the object-oriented view of a business system which represents the "causal" view of the Real World. Each removed part of the normalized process should be identified with some business system object. This object points to the general meaning of the new support process, created from the removed sub-process. The general meaning of this object can be subsequently used for the generalization of this new process in order to achieve its needed stability. For instance, processes such as Expedition\&Delivery, Payment Management, Production Planning, etc. from the Process Map in Figure 9, are typical representatives of standard processes which are absolutely independent of any specific key process which can use their services.

As its name signifies, the process normalization technique was originally inspired by the crucial work in the field of conceptual modeling [8]. In this work Peter Chen expressed the need for perceiving the data base of the organization as a model of a relevant part of the Real World in terms of objects (entities), their mutual associations (relationships) and classifications (types) which are nowadays known in informatics as a 'Principle of Modeling'. This principle should not be limited just to the field of data (i.e. Real World objects, Real World ontology) but regarded as a universal principle, valid even in the field of operations (i.e. business processes, Real World behavior). It is just this universality of the Principle of Modeling that uncovers the essential relationship between these two parts of the Real World: objects and processes, alias ontology of the Real World and behavior in the Real World. Looking at this relationship from a philosophical point of view, one can also see general consequences of the causality and the intentionality in Real World.

\section{Conclusions}

The purpose and practical meaning of the Process Normalization are similar to the technique for "normalization of data structures" [5], [7]. As in the case of the Data Normalization technique the main value of the Process Normalization does not lie in the procedure and its particular steps, in terms of the strict instruction for making decisions in the defined order. Once the user of this 
technique understands its essential meaning, he/she usually loses the ability to use it as a directive procedure, because he/she anticipates what should be the result of the next steps, which influences his/her way of thinking and prevents him/her from keeping the prescribed procedure. Instead of being a prescription for mechanically making decisions in a defined order, the Process Normalization rather serves as a 'training program' for understanding the essential meaning and purpose of the proper division of process steps into the natural process chains, related to existing business objects.

The main limitations of the Process Normalization technique are similar to the limitations of the Data Normalization. The technique itself is just a framework for the crucial decisions in the process of creating the conception of the system of processes. It cannot be used as a universal decision-making mechanism. Correctness and quality of each particular decision about the structure of the process system always depend mainly on the correct interpretation of the result and its consequences. Used just mechanically, the technique can easily lead to the senseless partitioning of processes up to the level close to the level of single activities. As with all aspects of process-driven management, the creation of the process system via partitioning of key processes is a complex, multidimensional task. So the particular decision about the contents of the given business process has always to be based on the meaning of the process in the context of available technology, market offer of standard services, existing technology framework, together with the level of knowledge in the organization, and, consequently, also on the overall ability of the organization to accept the future technology possibilities in terms of the organizational maturity, as it is explained in [15], for instance.

In the near future we expect possible development of successive process normal forms and also the analysis of relationships among the principles expressed in the Process Normalization, and some relevant aspects of the conceptual analysis; for instance, the problem of generalization structures in the conceptual model and its manifestation in the structure of supporting processes. Such reflections may also take the form of new succeeding normal forms.

All these visions of possible future development emphasize the essential meaning of the process normalization. It is not only the training procedure for understanding the essence of proper division of activities to processes, but also the framework for the generation of stimuli for the further research in the field of business process modeling and its relationships to other fields such as conceptual modeling, for instance.

\section{References}

[1] Object Management Group: Business Process Model and Notation (BPMN) Specification Version 2.0.2., Available: http://www.omg.org/spec/BPMN/

[2] Business System Modeling Specification, 2000-2016. Available: http://opensoul.panrepa.org/metamodel.html

[3] V. Ǩepa, "Normalization of Processes - Toward an Integrated View of Business Process System," in Proceedings of the BIR 2017 pre-BIR Forum, CEUR Workshop Proceedings, vol. 1898, 2017.

[4] M. Hammer and J. Champy, Reengineering the Corporation: A Manifesto for Business Revolution, London: Nicholas Brealey Publishing, 1993.

[5] E. F. Codd, "A Relational Model of Data for Large Shared Data Banks," Communications of the ACM, vol. 13, no. 6, pp. 377-387, 1970. Available: https://doi.org/10.1145/362384.362685

[6] E. F. Codd, "Recent Investigations into Relational Data Base Systems," in IBM Research Report, RJ1385, 1974.

[7] W. Kent, "A Simple Guide to Five Normal Forms in Relational Database Theory," Communications of the $A C M$, vol. 26, no. 2, pp. 120-125, 1983. Available: https://doi.org/10.1145/358024.358054

[8] P. P.-S.Chen, "The Entity-Relationship Model-Toward a Unified View of Data," ACM Transactions on Database Systems, vol. 1, no. 1, pp. 9-36, 1976. Available: https://doi.org/10.1145/320434.320440 
[9] T. H. Davenport, Process Innovation: Reengineering Work Through Information Technology, Harvard Business Review Press, 1992.

[10] M. A. Jackson, Principles of Program Design, Academic Press, London, UK, 1975.

[11] M. A. Jackson, System Development, Prentice Hall International Series in Computer Science, Prentice Hall, London, UK, 1983.

[12] V. Řepa, "Business Process Modeling Notation from the Methodical Perspective," in Towards a Service-Based Internet. ServiceWave 2010 Workshops. Lecture Notes in Computer Science, vol. 6569 Springer, pp. 160-171, 2011. Available: https://doi.org/10.1007/978-3-642-22760-8_18

[13] H. E. Eriksson and M. Penker, Business Modeling with UML: Business Patterns at Work, Wiley, 2000.

[14] T. H. Tse, "Integrating the structured analysis and design models: a Category-Theoretic Approach," Australian Computer Journal, vol. 19, no. 1, pp. 25-31, 1987.

[15] M. Hammer, “The Process Audit,” Harvard Business Review, April, 2007. 